\title{
Análisis bibliométrico sobre las publicaciones de eutanasia en SCOPUS, MEDLINE/Pubmed, Science Citation Index y SciELO
}

\author{
Bibliometric analysis of euthanasia publications in SCOPUS, MEDLINE / Pubmed, Science Citation Index \\ and SciELO
}

\section{Sr. Editor:}

Los estudios bibliométricos como rama de la cienciometría, nos permiten delimitar el estado actual de la investigación sobre cualquier aspecto en ciencias de la salud (1); en este caso se pretende evaluar el estado de las publicaciones científicas sobre eutanasia en todo el mundo, ésta etimológicamente significa "buena muerte", conocida como la manera ideal de morir, siendo un concepto sociocultural que se transforma, mientras que la Organización Mundial de la Salud (OMS) la define como la acción deliberada que realiza una persona con la intención de provocar la muerte sin dolor (2).

El objetivo de la eutanasia es aliviar el sufrimiento físico, emocional o espiritual incontrolable que puede estar cursando el paciente; este concepto se tiende a dividir en activa y pasiva, siendo la primera, legal en Holanda, Bélgica, Luxemburgo y en Colombia, mientras que la eutanasia pasiva es permitida por diversas regulaciones en Canadá, España, Inglaterra, India, entre otros, siendo esta una práctica ampliamente usada en Medicina intensiva a nivel mundial $(2,3)$.

En base a lo anterior, se realizó un estudio observacional, descriptivo, transversal de tipo bibliométrico sobre la producción científica mundial de la eutanasia en las bases de datos SCOPUS, MEDLINE/Pubmed (usando GoPubMed (C),Science Citation Index (SCI) y SciELO, el término utilizado fue "euthanasia" con su respectiva traducción al español. De cada base de datos se estudió el número de publicaciones, el país, los autores, el año, y el índice $H$. No se aplicó restricción en cuanto al tiempo.
Se encontraron 52961 artículos, 32575 artículos en SCOPUS (8598 de Estados Unidos, 2212 del Reino Unido y 1168 de Canadá), 1373 fueron publicados en el 2004, 1344 en 2005 y 1185 en 1996; los autores con mayor número de artículos fueron Van der Wal G con 147 artículos, Deliens L con 119 y Van der Heide A, con 118; el índice H desde 2007 al 2017 fue 161. En Medline se encontraron 14106 artículos (610 de Estados Unidos, 143 del Reino Unido y 105 de los Países bajos), 168 fueron publicados en 1996, 151 en 1993 y 146 en 1997; los autores más productivos fueron Van der Wal G 15 artículos, Onwuteaka-Philipsen B siete artículos, y Sheldon T seis artículos (Figura 1). En SCI fueron encontrados 5732 artículos (1891 de Estados Unidos, 540 de Brasil y 464 de Inglaterra), 638 fueron publicados en el 2016, 565 en 2015 y 526 en 2013; los autores más relevantes fueron Deliens L quien tenía 105 artículos, Nwuteaka-Philipsen BD 79 artículos y Van der Heide A 77 artículos; el índice H de eutanasia en esta base de datos fue 63 ( 7,17 promedio de citas por elemento). Por último, en SciELO se encontraron 548 artículos (366 de Brasil, 39 de Chile y 34 de Colombia), 54 se publicaron en el 2011, 48 en 2013, y 47 en el 2008.

Se puede apreciar una alta productividad científica sobre la eutanasia, un tema totalmente controversial a nivel mundial; esto implica el alto nivel de discusión o evidencia que puede existir sobre el tema. Es conveniente destacar el papel que Estados Unidos, Reino Unido y Canadá han desempeñado y demostrado en la investigación de la eutanasia, mientras que Latinoamérica tiene una producción científica muy baja, en donde Brasil es el único país que tiene un nivel aceptable.

1. Facultad de Ciencias Médicas, Universidad Nacional de Caaguazú. Cnel. Oviedo, Paraguay.

2. Facultad de Ciencias de la Salud, Universidad de Carabobo Sede Aragua. Aragua,Venezuela. 


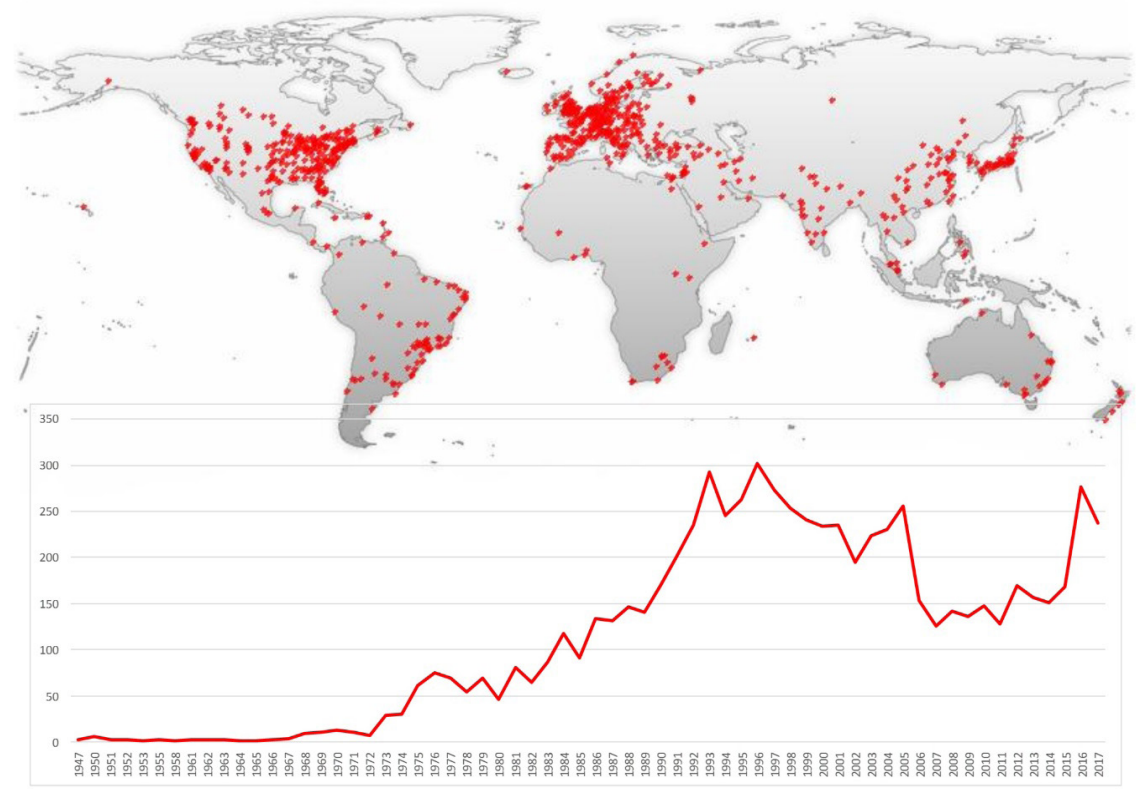

Figura 1. Distribución de las publicaciones científicas sobre eutanasia según país y año, 1947 a 2017

(Fuente: GoPubmed (C).

Es importante que los artículos encontrados sean sintetizados en guías, manuales, artículos de revisión o cualquier otro medio que permita que los médicos de diversos países logren delimitar si la eutanasia o no es una práctica recomendable, ya que en la actualidad el tratamiento de las enfermedades es más eficaz, debido a la mejora en la prevención y a los medicamentos existentes, pero difícilmente se evita la muerte, puesto que ningún avance ha sido lo suficientemente efectivo para poder superarla. La muerte no es el peor resultado posible, ya que a veces los médicos logran la supervivencia del paciente en condiciones que se pueden juzgar peor que la muerte, en donde es cuestionable el preservar la vida bajo cualquier circunstancia demostrando que la dignidad de la vida también cuenta como un factor a discutir cuando se enfrenta la muerte, pudiendo transformar los objetivos del médico de prevenir, curar y aliviar a ayudar a morir con dignidad $(4,5)$.

En conclusión, podemos apreciar una alta producción científica sobre eutanasia lo que denota el gran estudio en torno a este tema; sin embargo, es fundamental continuar debatiendo y lograr determinar si es una de las futuras prácticas que deberán ejercer los médicos tratantes en los distintos países.

\section{Carlos Miguel Rios-González ${ }^{1}$, Ginno Alessandro De Benedictis-Serrano ${ }^{2}$, Laura Valentina Contreras Lugo ${ }^{2}$, Génesys José Córdova-Rivas ${ }^{2}$, María Lucía Contreras Romero ${ }^{2}$.}

\section{Correspondencia:}

Carlos Miguel Rios González

Sargento Toribio Valinoti c/ Sgto. 1ro Lorenzo Ayala Rolon, 1721, Asunción, Paraguay.

Correo electrónico: carlosmigue_rios@live.com

Telefono:595971708328

\section{REFERENCIAS BIBLIOGRÁFICAS}

1. Araújo JA, Arencibia JR. Informetría, bibliometría y cienciometría: aspectos teórico-prácticos. Acimed. 2002; 10(4):5-6.

2. Mendoza-Villa JM, Herrera-Morales LA. Reflexiones acerca de la eutanasia en Colombia. RevColombAnestesiol. 2016; 44(4):324-329.

3. Carrasco M VH, Crispi F. Eutanasia en Chile: Una discusión pendiente. Ver Med Chile. 2016; 144(12):1598-1604.

4. Kessel H. Paradojas en las decisiones al final de la vida. Medicina Clínica.2001;116(8):296-298.

5. Gervas J. Morir en casa con dignidad. Una posibilidad, si hay apoyo y cuidados de calidad. Gaceta Médica de Bilbao. 2011;108(1):3-6

Recibido: 12/02/2018 